\title{
THE EXISTENCE OF QUERMASS-INTERACTION PROCESSES FOR NONLOCALLY STABLE INTERACTION AND NONBOUNDED CONVEX GRAINS
}

\author{
DAVID DEREUDRE, ${ }^{*}$ Université de Valenciennes et du Hainaut-Cambrésis
}

\begin{abstract}
We prove the existence of infinite-volume quermass-interaction processes in a general setting of nonlocally stable interaction and nonbounded convex grains. No condition on the parameters of the linear combination of the Minkowski functionals is assumed. The only condition is that the square of the random radius of the grain admits exponential moments for all orders. Our methods are based on entropy and large deviation tools.
\end{abstract}

Keywords: Stochastic geometry; Boolean model; germ-grain model; Gibbs point process; quermass-interaction process

2000 Mathematics Subject Classification: Primary 60D05; 82B21

\section{Introduction}

Physicists and biologists are often confronted with problems involving complex random sets. Micro-emulsions, porous media, liquid-vapor interface structures are all examples of random geometrical structures that must be studied and comprehended. One admissible model for these random structures is the Poisson Boolean model, which is a random set, unifying independent random convex sets that are uniformly distributed in space. This model has been abundantly studied (see, for example, [8] and [13]). Many authors (see [1], [5]-[7], and [13]) have mentioned the need of developing, via Gibbs measures, an interacting germ-grain model where the interaction would be a functional of the local geometry of the set. The first such models are the Widom-Rowlinson model introduced in [14] and afterward, the area-interaction process in [1], and the morphological model in [7]. Finally, Kendall et al. [5] proposed a unified general model called the quermass-interaction process, where the interaction is a linear combination of the fundamental Minkowski functionals (i.e. in $\mathbb{R}^{2}$, the area, the perimeter, and the Euler-Poincaré characteristic).

The existence results of infinite volume quermass-interaction processes in [1] and [5] are based on Preston's approach (see [11]), where the needed assumptions are either the local stability or the superstability. As the quermass-interaction is never superstable, the existence results are always for locally stable quermass-interactions, which is only the case for the area Minkowski functional under some conditions. So, even if very interesting results concerning the stability of the quermass-interaction have been developed in [5], they do not ensure the existence of infinite volume quermass-interaction processes in a general setting. Moreover, these existence results only concern the case of bounded grains. The case of nonbounded grains is a difficult problem in statistical mechanics because it induces nonlocal Gibbs kernels.

Received 19 September 2008; revision received 26 March 2009.

* Postal address: LAMAV, Université de Valenciennes et du Hainaut-Cambrésis, Le Mont Houy, 59313 Valenciennes Cedex 09, France. Email address: david.dereudre@univ-valenciennes.fr 
In this paper we prove the existence of infinite-volume quermass-interaction processes in a general setting of nonlocally stable interaction and nonbounded convex grains.

Our methods are based on entropy tools developed in [3]. The authors proposed an alternative proof for the existence of Gibbs measures to that given in [11] or [12]. The main tool is a result on relative compactness for a family of probability measures having a specific entropy uniformly bounded. In our paper we essentially follow the same scheme and the unique condition, needed for the entropy and large deviation tools, is that, for every $\alpha>0$,

$$
\int_{\mathbb{R}^{+}} \exp \left(\alpha R^{2}\right) Q(\mathrm{~d} R)<+\infty,
$$

where $Q$ is the distribution on $\mathbb{R}^{+}$of the radius of the ball in the reference Poisson Boolean model.

In Section 2 we give the notation and some elementary results. We prove some basic lemmas about the stability of the interaction and finally we give the existence theorem. Section 3 is devoted to the proof of this theorem. We conclude the paper in Section 4.

\section{Notation, definitions, and elementary results}

\subsection{State spaces}

We denote by $\varepsilon$ the space $\mathbb{R}^{2} \times \mathbb{R}^{+}$endowed with the natural Euclidean Borel $\sigma$-algebra $\sigma\left(\mathbb{R}^{2} \times \mathbb{R}^{+}\right)$and by $\mathcal{B}\left(\mathbb{R}^{2}\right)$ the set of bounded Borel sets in $\mathbb{R}^{2}$. We denote by $\mathcal{M}$ the set of the integer-valued measures $\gamma$ on $\&$ such that, for every $\Lambda$ in $B\left(\mathbb{R}^{2}\right), \gamma\left(\Lambda \times \mathbb{R}^{+}\right) \in \mathbb{N}$. It is endowed with the $\sigma$-algebra $\sigma(\mathcal{M})$ generated by the function $\gamma \mapsto \gamma\left(\Lambda \times \mathbb{R}^{+}\right)$. We denote by $\mathcal{M}_{f}$ the subspace of finite measures in $\mathcal{M}$. Any measure $\gamma \in \mathcal{M}$ has the following representation:

$$
\gamma=\sum_{i \in \mathcal{I}} \delta_{\left(x_{i}, R_{i}\right)}
$$

where $\mathcal{I}$ is a subset of $\mathbb{N},\left(x_{i}\right)_{i \in \mathcal{X}}$ are elements of $\mathbb{R}^{2}$, and $\left(R_{i}\right)_{i \in \mathcal{I}}$ are elements of $\mathbb{R}^{+}$. The marked points $\left(x_{i}, R_{i}\right)_{i \in \mathcal{X}}$ are not necessarily distinct. The configuration $\gamma$ may be seen as a marked integer-valued measure on $\mathbb{R}^{2}$ with marks in $\mathbb{R}^{+}$. The first coordinates of $\gamma$ denote the locations, while the second coordinates represent the marks.

We write $(x, R) \in \gamma$ if $\gamma(\{(x, R)\})>0$ and $x \in \gamma$ if $\gamma\left(\{x\} \times \mathbb{R}^{+}\right)>0$. For $\Lambda$ in $\mathbb{R}^{2}$, we note that $\gamma_{\Lambda}$ is the restriction of $\gamma$ on $\Lambda \times \mathbb{R}^{+}$, which is just the measure $\sum_{(x, R) \in \gamma \cap \Lambda \times \mathbb{R}^{+}} \delta_{(x, R)}$.

We consider a smaller space than $\mathcal{M}$ for the space of tempered configurations. For every $K \geq 1$ and $K^{\prime} \geq 0$, we define $\mathcal{M}_{K, K^{\prime}}$ to be the set of $\gamma$ in $\mathcal{M}$ such that, for every $n \geq 1$,

$$
\frac{1}{\pi n^{2}} \sum_{(x, R) \in \gamma_{B(0, n)}}\left(1+R^{2}\right) \leq K \quad \text { and } \quad \sup _{(x, R) \in \gamma_{B(0, n)}} R \leq \frac{1}{2} n+K^{\prime} .
$$

So the space of tempered configurations is defined by

$$
\mathcal{M}_{\mathcal{T}}=\bigcup_{K \geq 1, K^{\prime} \geq 0} \mathcal{M}_{K, K^{\prime}}
$$

We let $\mathcal{P}\left(\mathcal{M}_{\mathcal{T}}\right)$ be the space of probability measures on $\mathcal{M}_{\mathcal{T}}$.

The germ-grain set associated to $\gamma$ in $\mathcal{M}_{\mathcal{T}}$ is defined by

$$
\Gamma=\bigcup_{(x, R) \in \gamma} B(x, R),
$$

where $B(x, r)$ is the closed ball centered at $x$ with radius $R$. By the following lemma we can 
easily prove that every $\gamma$ in $\mathcal{M}_{\mathcal{T}}$ generates a locally finite germ-grain set $\Gamma$. It means that, for every $\Lambda$ in $\mathscr{B}\left(\mathbb{R}^{2}\right)$, there is only a finite number of balls in $\Gamma$ hitting $\Lambda$.

Lemma 2.1. Let $\gamma$ be in $\mathcal{M}_{K, K^{\prime}}\left(K \geq 1\right.$ and $\left.K^{\prime} \geq 0\right)$. Then, for every $R_{1}>0$ and every $(x, R)$ in $\gamma$ such that $|x| \geq 2\left(R_{1}+K^{\prime}+1\right)$, we have $B(x, R) \cap B\left(0, R_{1}\right)=\varnothing$.

Proof. Let $\gamma$ be in $\mathcal{M}_{K, K^{\prime}}$, and let $(x, R)$ be in $\gamma$ such that $|x| \geq 2\left(R_{1}+K^{\prime}+1\right)$. Then

$$
\begin{aligned}
|x|-R & \geq|x|-\frac{1}{2}([|x|]+1)-K^{\prime} \\
& \geq \frac{|x|}{2}-K^{\prime}-\frac{1}{2} \\
& \geq R_{1}+\frac{1}{2} \\
& >R_{1},
\end{aligned}
$$

where $[|x|]$ denotes the integer part of $|x|$. So, the intersection of the balls $B\left(0, R_{1}\right)$ and $B(x, R)$ is empty.

\subsection{Interaction}

We define the quermass-interaction energy as in [5]. Let us begin with the energy of the finite configurations. Throughout the paper, $\theta_{1}, \theta_{2}$, and $\theta_{3}$ are three fixed real numbers. They may be positive or not.

Definition 2.1. For every $\gamma$ in $\mathcal{M}_{f}$, we define the energy of $\gamma$ by

$$
H(\gamma)=\left(\theta_{1} \mathcal{A}+\theta_{2} \mathcal{L}+\theta_{3} \chi\right)\left(\bigcup_{(x, R) \in \gamma} B(x, R)\right)
$$

where $\mathcal{A}, \mathcal{L}$, and $\chi$ are the three fundamental Minkowski functionals: area, perimeter, and the Euler-Poincaré characteristic.

See [5] for more details about the functionals $\mathcal{A}, \mathcal{L}$, and $\chi$. Let us remark that the energy of a finite configuration is always defined. However, it is not easy to define the local energy of an infinite configuration inside a finite window $\Lambda$. If the germ-grain model associated to the configuration $\gamma$ is not local, the energy may be not defined. The restriction to the space of tempered configurations is needed.

Definition 2.2. Let $\Lambda$ be in $\mathscr{B}\left(\mathbb{R}^{2}\right)$, and let $\gamma$ be in $\mathcal{M}_{\mathcal{T}}$. We define the energy of $\gamma$ inside $\Lambda$ by the following limit:

$$
H_{\Lambda}(\gamma)=\lim _{\Delta \rightarrow \mathbb{R}^{2}}\left[H\left(\gamma_{\Delta}\right)-H\left(\gamma_{\Delta \backslash \Lambda}\right)\right],
$$

where $\Delta \backslash \Lambda$ is the set $\{x \in \Delta$ such that $x \notin \Lambda\}$.

Let us prove that the limit defined above is always well defined. Let us show that, for large enough $\Delta$, the difference $H\left(\gamma_{\Delta}\right)-H\left(\gamma_{\Delta \backslash \Lambda}\right)$ is constant. Since $\gamma$ is in $\mathcal{M}_{\mathcal{T}}$, the configuration $\gamma$ is in $\mathcal{M}_{K, K^{\prime}}$ for some $K \geq 1$ and $K^{\prime} \geq 0$. There exists $R_{1}$ such that $\Lambda$ is included in $B\left(0, R_{1}\right)$. Let $R_{2}$ equal $\frac{3}{2} R_{1}+K^{\prime}+\frac{1}{2}$. So, thanks to definition (2.1), for every $(x, R) \in \gamma_{\Lambda}, B(x, R)$ is included in $B\left(0, R_{2}\right)$. By Lemma 2.1 , for a bounded set $\Delta$ containing $B\left(0,2\left(R_{2}+K^{\prime}+1\right)\right)$, we find that, for every $\left(x^{\prime}, R^{\prime}\right) \in \gamma_{\Delta^{\mathrm{c}}}$, the balls $B(x, R)$ and $B\left(x^{\prime}, R^{\prime}\right)$ are disjoint. Therefore, 
noting that $\Lambda$ is included in $\Delta$ and from the additivity of the Minkowski functionals,

$$
\begin{aligned}
H\left(\gamma_{\Delta}\right) & -H\left(\gamma_{\Delta \backslash \Lambda}\right) \\
= & W\left(\bigcup_{(x, R) \in \gamma_{\Delta}} B(x, R)\right)-W\left(\bigcup_{(x, R) \in \gamma_{\Delta \backslash \Lambda}} B(x, R)\right) \\
= & W\left(\bigcup_{(x, R) \in \gamma_{\Lambda}} B(x, R)\right)-W\left(\left(\bigcup_{(x, R) \in \gamma_{\Lambda}} B(x, R)\right) \cap\left(\bigcup_{(x, R) \in \gamma_{\Delta \backslash \Lambda}} B(x, R)\right)\right) \\
= & \left.W\left(\bigcup_{\substack{(x, R) \in \gamma_{\Lambda} \\
\left(x^{\prime}, R^{\prime}\right) \in \gamma_{B\left(0,2\left(R_{2}+K^{\prime}\right)\right) \backslash \Lambda}}} B(x, R)\right)-W(x, R) \cap B\left(x^{\prime}, R^{\prime}\right)\right),
\end{aligned}
$$

where $W$ is the additive functional $\theta_{1} \mathcal{A}+\theta_{2} \mathcal{L}+\theta_{3} \chi$. The last equality shows that $H\left(\gamma_{\Delta}\right)-$ $H\left(\gamma_{\Delta \backslash \Lambda}\right)$ does not depend on $\Delta$ being large enough.

In the following lemma we prove the additivity of the energy $H_{\Lambda}$, which will be needed to satisfy the compatibility of the Gibbs kernels defined in (2.2), below.

Lemma 2.2. Let $\Lambda \subset \Lambda^{\prime}$ be sets in $\mathcal{B}\left(\mathbb{R}^{2}\right)$. Then, there exists a function $\varphi_{\Lambda, \Lambda^{\prime}}$ from $\mathcal{M}_{\mathcal{T}}$ to $\mathbb{R}$ such that, for every $\gamma$ in $\mathcal{M}_{\mathcal{T}}$,

$$
H_{\Lambda^{\prime}}(\gamma)=H_{\Lambda}(\gamma)+\varphi_{\Lambda, \Lambda^{\prime}}\left(\gamma_{\Lambda^{c}}\right)
$$

Proof. Let $\Lambda \subset \Lambda^{\prime}$ be sets in $\mathcal{B}\left(\mathbb{R}^{2}\right)$, and let $\gamma$ be in $\mathcal{M}_{\mathcal{T}}$. Then

$$
\begin{aligned}
H_{\Lambda^{\prime}}(\gamma) & =\lim _{\Delta \rightarrow \mathbb{R}^{2}}\left[H\left(\gamma_{\Delta}\right)-H\left(\gamma_{\Delta \backslash \Lambda^{\prime}}\right)\right] \\
& =\lim _{\Delta \rightarrow \mathbb{R}^{2}}\left[H\left(\gamma_{\Delta}\right)-H\left(\gamma_{\Delta \backslash \Lambda}\right)\right]+\lim _{\Delta \rightarrow \mathbb{R}^{2}}\left[H\left(\gamma_{\Delta \backslash \Lambda}\right)-H\left(\gamma_{\Delta \backslash \Lambda^{\prime}}\right)\right] \\
& =H_{\Lambda}(\gamma)+\varphi_{\Lambda, \Lambda^{\prime}}\left(\gamma_{\Lambda^{c}}\right) .
\end{aligned}
$$

Now, let us show some stability properties for the energy.

Lemma 2.3. There exists a constant $C$ such that, for every $\gamma$ in $\mathcal{M}_{f}$,

$$
|H(\gamma)| \leq C\left(\sum_{(x, R) \in \gamma}\left(1+R^{2}\right)\right) .
$$

Proof. This result is obvious for the part of the energy coming from the functionals $\mathcal{A}$ and $\mathcal{L}$. Concerning the contribution of $\chi$, Kendall et al. [5, Theorem 4.3] proved that the Euler-Poincaré characteristic $\chi$ of a union of $n$ balls is bounded from above by $3 n-5$.

It is more difficult to prove the stability of the local energy $H_{\Lambda}$.

Lemma 2.4. Let $\Lambda$ be a set in $\mathcal{B}\left(\mathbb{R}^{2}\right)$. Let $K \geq 1$ and $K^{\prime} \geq 0$ be constants. Then, there exists a constant $C^{\prime}$ such that, for every $\gamma^{\prime}$ in $\mathcal{M}$ and every $\gamma$ in $\mathcal{M}_{K, K^{\prime}}$,

$$
\left|H_{\Lambda}\left(\gamma_{\Lambda}^{\prime}+\gamma_{\Lambda^{c}}\right)\right| \leq C^{\prime} \sum_{(x, R) \in \gamma_{\Lambda}^{\prime}}\left(1+R^{2}\right) .
$$


Proof. Let $\Lambda$ be a set in $\mathcal{B}\left(\mathbb{R}^{2}\right)$, and let $K \geq 1$ and $K^{\prime} \geq 0$ be constants. Let $\gamma^{\prime}$ be in $\mathcal{M}$ and $\gamma$ be in $\mathcal{M}_{K, K^{\prime}}$. There exists $R_{1}>0$ such that $\Lambda \subset B\left(0, R_{1}\right)$, and we define

$$
\bar{R}=\max _{(x, R) \in \gamma_{\Lambda}^{\prime}} R .
$$

Thanks to Lemma 2.1, putting $\Delta=B\left(0,2\left(R_{1}+\bar{R}+K^{\prime}+1\right)\right)$, we have $B(x, R) \cap B\left(x^{\prime}, R^{\prime}\right)=\varnothing$ for all $(x, R) \in \gamma_{\Delta^{\mathrm{c}}}$ and all $\left(x^{\prime}, R^{\prime}\right) \in \gamma_{\Lambda}^{\prime}$. So,

$$
\left|H_{\Lambda}\left(\gamma_{\Lambda}^{\prime}+\gamma_{\Lambda^{c}}\right)\right|=\left|H\left(\gamma_{\Lambda}^{\prime}+\gamma_{\Delta \backslash \Lambda}\right)-H\left(\gamma_{\Delta \backslash \Lambda}\right)\right|
$$

From Lemma 2.3 and the properties in (2.1),

$$
\begin{aligned}
\left|H_{\Lambda}\left(\gamma_{\Lambda}^{\prime}+\gamma_{\Lambda^{c}}\right)\right| & \leq C \sum_{(x, R) \in \gamma_{\Lambda}^{\prime}+\gamma_{\Delta \backslash \Lambda}}\left(1+R^{2}\right)+C \sum_{(x, R) \in \gamma_{\Delta \backslash \Lambda}}\left(1+R^{2}\right) \\
& \leq C \sum_{(x, R) \in \gamma_{\Lambda}^{\prime}}\left(1+R^{2}\right)+2 C \sum_{\substack{(x, R) \in \gamma \\
|x| \leq 2\left(R_{1}+\bar{R}+K^{\prime}+1\right)}}\left(1+R^{2}\right) \\
& \leq C \sum_{(x, R) \in \gamma_{\Lambda}^{\prime}}\left(1+R^{2}\right)+2 C K \pi\left(2\left(R_{1}+\bar{R}+K^{\prime}+1\right)+1\right)^{2} \\
& \leq C^{\prime} \sum_{(x, R) \in \gamma_{\Lambda}^{\prime}}\left(1+R^{2}\right) .
\end{aligned}
$$

The last inequality is due to the fact that $\bar{R}$ is equal to one of the radii of the $(x, R)$ points in $\gamma_{\Lambda}^{\prime}$. Obviously, $C^{\prime}$ depends on $\Lambda, K$, and $K^{\prime}$.

\subsection{Quermass-interaction process: the Gibbs property}

Denote by $\lambda$ the Lebesgue measure on $\mathbb{R}^{2}$, and let $Q$ be a probability measure on $\mathbb{R}^{+}$. Suppose that $\pi$ stands for the Poisson process on $\mathscr{E}$ with intensity measure $z \lambda \otimes Q(z>0)$, where $Q$ is the reference measure for the marks of the point process. For every $\Lambda \in \mathscr{B}\left(\mathbb{R}^{2}\right)$, $\pi_{\Lambda}$ denotes the Poisson process on $\Lambda \times \mathbb{R}^{+}$with intensity measure $z \lambda_{\Lambda} \otimes Q$. We assume the following assumption for the probability measure $Q$.

(H) For all $\alpha \geq 0$,

$$
\int_{\mathbb{R}^{+}} \exp \left(\alpha R^{2}\right) Q(\mathrm{~d} R)<+\infty .
$$

In [9], the authors showed that the minimal assumption on $Q$, to define kernels (2.2) in the following definition, is assumption $(\mathrm{H})$ with the constant $\alpha$ equal to $-\theta_{1} \pi$ (if $\theta_{1}<0$ ). In our paper we need more to prove the existence of infinite Gibbs measures. In fact, only assumption $(\mathrm{H})$ will be needed to apply some entropy and large deviation tools.

Now, let us introduce the Gibbs kernels.

Definition 2.3. For every $\Lambda$ in $\mathcal{B}\left(\mathbb{R}^{2}\right)$, the kernel $\Xi_{\Lambda}$ is a function from $\mathcal{M}_{\mathcal{T}} \times \mathcal{P}\left(\mathcal{M}_{\mathcal{T}}\right)$ to $\mathbb{R}$ defined as follows: for every $\gamma \in \mathcal{M}_{\mathcal{T}}$ and every bounded positive measurable function $f$ from $\mathcal{M}_{\mathcal{T}}$ to $\mathbb{R}$,

$$
\int_{\mathcal{M}_{\mathcal{T}}} f\left(\gamma^{\prime}\right) \Xi_{\Lambda}\left(\gamma, \mathrm{d} \gamma^{\prime}\right)=\int_{\mathcal{M}} f\left(\gamma_{\Lambda}^{\prime}+\gamma_{\Lambda^{\mathrm{c}}}\right) \frac{1}{Z_{\Lambda}\left(\gamma_{\Lambda^{\mathrm{c}}}\right)} \exp \left(-H_{\Lambda}\left(\gamma_{\Lambda}^{\prime}+\gamma_{\Lambda^{\mathrm{c}}}\right)\right) \pi_{\Lambda}\left(\mathrm{d} \gamma_{\Lambda}^{\prime}\right),
$$


where

$$
Z_{\Lambda}\left(\gamma_{\Lambda^{c}}\right)=\int_{\mathcal{M}} \exp \left(-H_{\Lambda}\left(\gamma_{\Lambda}^{\prime}+\gamma_{\Lambda^{c}}\right)\right) \pi_{\Lambda}\left(\mathrm{d} \gamma_{\Lambda}^{\prime}\right) .
$$

Let us remark that the kernel $\Xi_{\Lambda}$ is well defined since, by Lemma 2.4 and assumption $(\mathrm{H})$, for every $\gamma$ in $\mathcal{M}_{\mathcal{T}}$,

$$
\begin{aligned}
Z_{\Lambda}\left(\gamma_{\Lambda^{\mathrm{c}}}\right) & \leq \int_{\mathcal{M}} \exp \left(C^{\prime} \sum_{(x, R) \in \gamma_{\Lambda}^{\prime}}\left(1+R^{2}\right)\right) \pi_{\Lambda}\left(\mathrm{d} \gamma_{\Lambda}^{\prime}\right) \\
& \leq \mathrm{e}^{-z|\Lambda|} \sum_{k=0}^{+\infty} \frac{1}{k !}\left(\mathrm{e}^{C^{\prime}}\right)^{k}(z|\Lambda|)^{k}\left(\int_{\mathbb{R}^{+}} \exp \left(C^{\prime} R^{2}\right) Q(\mathrm{~d} R)\right)^{k} \\
& \leq \mathrm{e}^{-z|\Lambda|} \exp \left(\mathrm{e}^{C^{\prime}} z|\Lambda| \int_{\mathbb{R}^{+}} \exp \left(C^{\prime} R^{2}\right) Q(\mathrm{~d} R)\right) \\
& <+\infty
\end{aligned}
$$

Now, we are able to give the definition of the quermass-interaction process.

Definition 2.4. ([5].) A probability measure $\mu$ in $\mathcal{P}\left(\mathcal{M}_{\mathcal{T}}\right)$ is a quermass-interaction process if, for every $\Lambda$ in $\mathscr{B}\left(\mathbb{R}^{2}\right)$ and every bounded positive measurable function $f$ from $\mathcal{M}_{\mathcal{T}}$ to $\mathbb{R}$,

$$
\int_{\mathcal{M}_{\mathcal{T}}} f(\gamma) \mu(\mathrm{d} \gamma)=\int_{\mathcal{M}_{\mathcal{T}}} \int_{\mathcal{M}_{\mathcal{T}}} f\left(\gamma^{\prime}\right) \Xi_{\Lambda}\left(\gamma, \mathrm{d} \gamma^{\prime}\right) \mu(\mathrm{d} \gamma) .
$$

These equations for all $\Lambda$ are called DLR (Dobrushin, Landford, Ruelle) equations.

See [11] for a general presentation of Gibbs measures and DLR equations. Our result is the following.

Theorem 2.1. Under assumption $(H)$, for every $\theta_{1}, \theta_{2}$, and $\theta_{3}$ in $\mathbb{R}$, there exists a quermassinteraction process.

\section{Proof of Theorem 2.1}

\subsection{Sketch of the proof}

In Section 3.2 we define a family of finite-volume Gibbs measures $\left(\hat{\mu}_{n}\right)$ on $[-n, n]^{2}$ (see (3.1), below), and the aim is to prove that these measures converge to a measure $\hat{\mu}$ which will be the infinite-volume Gibbs measure we seek. The topology used here is the weak topology induced by the local and tame functions on $\mathcal{M}$. Georgii and Zessin [4] proved that a set of measures is relatively compact if and only if the specific entropy of these measures is uniformly bounded (see Proposition 3.2, below). Therefore, we show via the fundamental Proposition 3.3, below, that the specific entropy of the measures $\left(\hat{\mu}_{n}\right)$ is uniformly bounded and we obtain the convergence of a subsequence $\left(\hat{\mu}_{\varphi(n)}\right)$ to a measure $\hat{\mu}$.

In Section 3.3 we study some properties of the measures $\hat{\mu}$ and $\left(\hat{\mu}_{n}\right)$. First of all, Lemma 3.2, below, implies that, $\hat{\mu}$-almost surely, the configuration $\gamma$ is in $\mathcal{M}_{\mathcal{T}}$. Moreover, to prove that $\hat{\mu}$ satisfies the DLR equations in the following section, we need a uniform property for the supports of measures $\left(\hat{\mu}_{n}\right)$. So, we show in Lemma 3.3, below, that, for every $\varepsilon>0$, there exists $K^{\prime}$ large enough such that, for every $n \geq 1, \hat{\mu}_{n}\left(\mathcal{M}_{K^{\prime}}\right) \geq 1-\varepsilon$, where $\mathcal{M}_{K^{\prime}}$ is defined in (3.8), below, and is equal to $\mathcal{M}_{K^{\prime},+\infty}$. 
In Section 3.4 we prove precisely that the measures $\hat{\mu}$ satisfy the DLR equations, (2.3). This part is based on some approximation techniques inspired by Preston's results in [11]. In general, we have to prove that the finite-volume Gibbs measures satisfy the DLR equations and we pass the limit through them to obtain the DLR equations for the limiting measure. Unfortunately, the measures $\left(\hat{\mu}_{n}\right)$ do not satisfy the DLR equations since they are the stationary periodic Gibbs measures. So we introduce the measures $\left(\bar{\mu}_{n}\right)$ (see (3.13), below), which are asymptotically equivalent to $\left(\hat{\mu}_{n}\right)$ and which satisfy the DLR equations. Afterward, via some technical approximations, we are able to pass the limit through the DLR equations satisfied by $\left(\bar{\mu}_{n}\right)$ and we obtain the DLR equations for $\hat{\mu}$.

\subsection{Convergence of the sequence $\left(\hat{\mu}_{n}\right)$}

Let $n \geq 1$. We set $\Lambda_{n}=[-n, n]^{2}$, and the finite-volume Gibbs measure $\mu_{n}$ on $\Lambda_{n}$ is defined by

$$
\mu_{n}(\mathrm{~d} \gamma)=\frac{1}{Z_{n}} \mathrm{e}^{-H(\gamma)} \pi_{\Lambda_{n}}(\mathrm{~d} \gamma)
$$

where

$$
Z_{n}=\int \mathrm{e}^{-H(\gamma)} \pi_{\Lambda_{n}}(\mathrm{~d} \gamma)
$$

By Lemma 2.3, $\mu_{n}$ is well defined since $Z_{n}<+\infty$.

We need to define a stationary finite-volume Gibbs measure $\hat{\mu}_{n}$ as in [3]. First of all, $\tilde{\mu}_{n}$ denotes the measure on $\mathcal{M}_{\mathcal{T}}$ relative to which the configurations in the disjoint blocks $\Lambda_{n}+(2 n+1) i, i \in \mathbb{Z}^{2}$, are independent with identical distribution $\mu_{n}$. Let $I_{n}$ be the set $\Lambda_{n} \cap \mathbb{Z}^{2}$, and let

$$
\hat{\mu}_{n}=\frac{1}{(2 n+1)^{2}} \sum_{i \in I_{n}} \tilde{\mu}_{n} \circ \vartheta_{i}^{-1}
$$

where $\vartheta_{i}$ is the translation on $\mathbb{R}^{2}$ or $\mathcal{M}\left(\mathbb{R}^{2}\right)$ by the vector $i$ in $\mathbb{Z}^{2}$ and $\tilde{\mu}_{n} \circ \vartheta_{i}^{-1}$ is the probability measure defined by

$$
\tilde{\mu}_{n} \circ \vartheta_{i}^{-1}(A)=\tilde{\mu}_{n}\left(\left\{\gamma \in \mathcal{M}, \vartheta_{i}(\gamma) \in A\right\}\right) \text { for all } A \in \sigma(\mathcal{M}) .
$$

Let us remark that $\hat{\mu}_{n}$ is invariant under the translations $\left(\vartheta_{i}\right)_{i \in \mathbb{Z}^{2}}$. Our aim is to obtain the convergence of $\hat{\mu}_{n}$ to the Gibbs measure $\mu$. So let us define precisely the convergence topology that we use.

A function $f$ is called local and tame if there exists $\Lambda$ in $\mathscr{B}\left(\mathbb{R}^{2}\right)$ and $A>0$ such that, for every $\gamma$ in $\mathcal{M}$,

$$
f(\gamma)=f\left(\gamma_{\Lambda}\right) \quad \text { and } \quad|f(\gamma)| \leq A\left(1+\sum_{(x, R) \in \gamma_{\Lambda}}\left(1+R^{2}\right)\right) .
$$

We denote by $\mathcal{W}$ the set of local and tame functions from $\mathcal{M}$ to $\mathbb{R}$. The topology $\tau_{\mathcal{W}}$ of local convergence on $\mathcal{P}(\mathcal{M})$ is then defined as the weak* topology induced by $\mathcal{W}$, i.e. as the smallest topology for which the mappings $P \mapsto \int f \mathrm{~d} P$ (with $f \in \mathcal{W}$ ) are continuous.

Now, let us define the specific entropy which is our main tool. Let $\mu$ and $\nu$ be probability measures in $\mathcal{P}(\mathcal{M})$. The relative entropy of $\mu$ with respect to $v$ on the set $\Lambda_{n}$ is defined by

$$
\tau_{\Lambda_{n}}(\mu \mid v)= \begin{cases}\int f \ln (f) \mathrm{d} v_{\Lambda_{n}} & \text { if } \mu_{\Lambda_{n}} \preceq v_{\Lambda_{n}}, \text { with } f=\mathrm{d} \mu_{\Lambda_{n}} / \mathrm{d} \nu_{\Lambda_{n}}, \\ +\infty & \text { otherwise, }\end{cases}
$$


where $\mu_{\Lambda_{n}} \preceq v_{\Lambda_{n}}$ means that $\mu_{\Lambda_{n}}$ is absolutely continuous with respect to $v_{\Lambda_{n}}$. The specific entropy of $\mu$ with respect to $v$ is defined by

$$
\mathcal{I}(\mu \mid v)=\lim _{n \rightarrow+\infty} \frac{1}{\left|\Lambda_{n}\right|} \tau_{\Lambda_{n}}(\mu \mid v) .
$$

The specific entropy is always defined and we have the following proposition (see [2] for a general presentation).

Proposition 3.1. Let $\mu$ and $v$ be probability measures in $\mathcal{P}(\mathcal{M})$. We have

(i) the application $\mu \mapsto \mathcal{I}(\mu \mid v)$ is affine,

(ii) the application $n \mapsto\left(1 /\left|\Lambda_{n}\right|\right) \mathcal{I}_{\Lambda_{n}}(\mu \mid \nu)$ is increasing.

Now, in the following we choose $v$ to equal $\pi$. So, we define

$$
\mathcal{I}(\mu)=\mathscr{I}(\mu \mid \pi) .
$$

We have the following fundamental proposition due to Georgii and Zessin [4].

Proposition 3.2. ([4, Proposition 2.6].) For every $A>0$, the set

$$
\{\mu \in \mathcal{P}(\mathcal{M}) \mid \mathcal{I}(\mu) \leq A\}
$$

is relatively compact for the topology $\tau_{w}$.

Let us remark that Assumption 2.1 of [4] with the function $\psi: R \mapsto 1+R^{2}$ is exactly our assumption (H). So, it plays a fundamental role at this part of the proof of Theorem 2.1.

By Proposition 3.2, to ensure that the set $\left(\hat{\mu}_{n}\right)$ is relatively compact, we just have to prove a uniform boundedness of the specific entropy. We have

$$
\begin{aligned}
\mathcal{I}\left(\hat{\mu}_{n}\right) & =\frac{1}{(2 n+1)^{2}} \sum_{i \in I_{n}} \mathcal{I}\left(\tilde{\mu}_{n} \circ \vartheta_{i}^{-1}\right) \\
& =\mathcal{I}\left(\tilde{\mu}_{n}\right) \\
& =\lim _{m \rightarrow+\infty} \frac{1}{\left|(2 m+1) \Lambda_{n}\right|} I_{(2 m+1) \Lambda_{n}}\left(\tilde{\mu}_{n} \mid \pi\right) \\
& =\lim _{m \rightarrow+\infty} \frac{1}{(2 m+1)^{2}\left|\Lambda_{n}\right|}(2 m+1)^{2} \tau_{\Lambda_{n}}\left(\mu_{n} \mid \pi\right) \\
& =\frac{1}{\left|\Lambda_{n}\right|} I_{\Lambda_{n}}\left(\mu_{n} \mid \pi\right) .
\end{aligned}
$$

From (3.2), we show in Proposition 3.3, below, that the uniform boundedness of the specific entropy is equivalent to that of the relative entropy of $\mu_{n}$ divided by the volume of $\Lambda_{n}$.

Proposition 3.3. There exists a constant $A>0$ such that, for every $n \geq 1$,

$$
\tau_{\Lambda_{n}}\left(\mu_{n} \mid \pi_{\Lambda_{n}}\right) \leq A\left|\Lambda_{n}\right|
$$

Before giving the proof, we need the following elementary large deviation lemma. 
Lemma 3.1. Let $\left(X_{n}\right)_{n \in \mathbb{N}^{*}}$ be a set of independent, identically distributed random variables such that, for every $\theta \in \mathbb{R}, L(\theta)=\mathrm{E}\left(\exp \left(\theta X_{1}\right)\right)$ is finite. Then, setting $S_{n}=\sum_{k=1}^{n} X_{k}$, we have, for every $a>\mathrm{E}\left(X_{1}\right)$,

$$
\mathrm{P}\left(\frac{S_{n}}{n} \geq a\right) \leq \mathrm{e}^{-n L^{*}(a)},
$$

where $L^{*}$ is the Cramer transform of $L$.

We know that the function $x \mapsto L^{*}(x)$ is a convex function from $\mathbb{R}$ to $\mathbb{R}$ which is null at $x=\mathrm{E}\left(X_{1}\right)$ and goes to $\infty$ when $x$ goes to $\infty$.

Proof of Proposition 3.3. We have

$$
\tau_{\Lambda_{n}}\left(\mu_{n} \mid \pi_{\Lambda_{n}}\right)=-\int H(\gamma) \mu_{n}(\mathrm{~d} \gamma)-\ln \left(Z_{n}\right) .
$$

Since $Z_{n} \geq \exp \left(-z\left|\Lambda_{n}\right|\right)$, we deduce by Lemma 2.3 that

$$
\tau_{\Lambda_{n}}\left(\mu_{n} \mid \pi_{\Lambda_{n}}\right) \leq C \int \sum_{(x, R) \in \gamma}\left(1+R^{2}\right) \mu_{n}(\mathrm{~d} \gamma)+z\left|\Lambda_{n}\right| .
$$

It remains to prove that

$$
\int \sum_{(x, R) \in \gamma}\left(1+R^{2}\right) \mu_{n}(\mathrm{~d} \gamma)=I_{1}+I_{2}+I_{3} \leq \text { constant }\left|\Lambda_{n}\right|,
$$

where

$$
\begin{gathered}
I_{1}=\int \mathbf{1}_{\left\{\sum_{(x, R) \in \gamma}\left(1+R^{2}\right) \leq A_{1}\left|\Lambda_{n}\right|\right\}} \sum_{(x, R) \in \gamma}\left(1+R^{2}\right) \mu_{n}(\mathrm{~d} \gamma), \\
I_{2}=\int \mathbf{1}_{\left\{\sum_{(x, R) \in \gamma}\left(1+R^{2}\right)>A_{1}\left|\Lambda_{n}\right|, \operatorname{card}(\gamma)>A_{2}\left|\Lambda_{n}\right|\right\}} \sum_{(x, R) \in \gamma}\left(1+R^{2}\right) \mu_{n}(\mathrm{~d} \gamma), \\
I_{3}=\int \mathbf{1}_{\left\{\sum_{(x, R) \in \gamma}\left(1+R^{2}\right)>A_{1}\left|\Lambda_{n}\right|, \operatorname{card}(\gamma) \leq A_{2}\left|\Lambda_{n}\right|\right\}} \sum_{(x, R) \in \gamma}\left(1+R^{2}\right) \mu_{n}(\mathrm{~d} \gamma) .
\end{gathered}
$$

The constants $A_{1}$ and $A_{2}$ will be fixed later.

There is no problem with the first term $I_{1}$ since $I_{1} \leq A_{1}\left|\Lambda_{n}\right|$. By Lemma 2.3,

$$
\begin{aligned}
& I_{2} \leq \int \mathbf{1}_{\left\{\operatorname{card}(\gamma)>A_{2}\left|\Lambda_{n}\right|\right\}} \sum_{(x, R) \in \gamma}\left(1+R^{2}\right) \mu_{n}(\mathrm{~d} \gamma) \\
& \leq \frac{1}{Z_{n}} \sum_{k=A_{2}\left|\Lambda_{n}\right|}^{+\infty} \mathrm{e}^{-z\left|\Lambda_{n}\right|} \frac{1}{k !}\left(z\left|\Lambda_{n}\right|\right)^{k} \int \cdots \\
& \quad \times \int \exp \left(C \sum_{i=1}^{k}\left(1+R_{i}^{2}\right)\right) \sum_{i=1}^{k}\left(1+R_{i}^{2}\right) Q\left(\mathrm{~d} R_{1}\right) \cdots Q\left(\mathrm{~d} R_{k}\right) \\
& \leq \mathrm{e}^{z\left|\Lambda_{n}\right|} \sum_{k=A_{2}\left|\Lambda_{n}\right|}^{+\infty} \mathrm{e}^{-z\left|\Lambda_{n}\right|} \frac{1}{k !}\left(z\left|\Lambda_{n}\right|\right)^{k} k \int\left(1+R^{2}\right) \exp \left(C\left(1+R^{2}\right)\right) Q(\mathrm{~d} R) \\
& \quad \times\left(\int \exp \left(C\left(1+R^{2}\right)\right) Q(\mathrm{~d} R)\right)^{k-1} .
\end{aligned}
$$


Thanks to assumption $(\mathrm{H})$, there exists a constant $B_{1}$ such that

$$
I_{2} \leq \sum_{k=A_{2}\left|\Lambda_{n}\right|}^{+\infty} \frac{1}{k !}\left(z\left|\Lambda_{n}\right|\right)^{k} B_{1}^{k} \leq \exp \left(z B_{1}\left|\Lambda_{n}\right|\right) \mathrm{P}\left(S_{\left|\Lambda_{n}\right|} \geq A_{2}\left|\Lambda_{n}\right|\right),
$$

where $\left(S_{m}\right)_{m \in \mathbb{N}^{*}}$ is a set of random variables distributed by a Poisson law with parameter $z B_{1} m$.

By Lemma 3.1 we choose $A_{2}$ large enough such that $L^{*}\left(A_{2}\right) \geq z B_{1}$, and we obtain

$$
I_{2} \leq \exp \left(z B_{1}\left|\Lambda_{n}\right|\right) \exp \left(-z B_{1}\left|\Lambda_{n}\right|\right) \leq 1 .
$$

For $I_{3}$, we have

$$
\begin{aligned}
& I_{3} \leq \frac{1}{Z_{n}} \sum_{k=0}^{A_{2}\left|\Lambda_{n}\right|} \mathrm{e}^{-z\left|\Lambda_{n}\right|} \frac{1}{k !}\left(z\left|\Lambda_{n}\right|\right)^{k} \int \cdots \int \mathbf{1}_{\left\{\sum_{i=1}^{k}\left(1+R_{i}^{2}\right)>A_{1}\left|\Lambda_{n}\right|\right\}} \exp \left(C \sum_{i=1}^{k}\left(1+R_{i}^{2}\right)\right) \\
& \times \sum_{i=1}^{k}\left(1+R_{i}^{2}\right) Q\left(\mathrm{~d} R_{1}\right) \cdots Q\left(\mathrm{~d} R_{k}\right) \\
& \leq \sum_{k=0}^{A_{2}\left|\Lambda_{n}\right|} \frac{1}{k !}\left(z\left|\Lambda_{n}\right|\right)^{k}\left(\int \cdots \int \mathbf{1}_{\left\{\sum_{i=1}^{k}\left(1+R_{i}^{2}\right)>A_{1}\left|\Lambda_{n}\right|\right\}} Q\left(\mathrm{~d} R_{1}\right) \cdots Q\left(\mathrm{~d} R_{k}\right)\right)^{1 / 2} \\
& \times\left(\int \cdots \int \exp \left(2 C \sum_{i=1}^{k}\left(1+R_{i}^{2}\right)\right)\left(\sum_{i=1}^{k}\left(1+R_{i}^{2}\right)\right)^{2} Q\left(\mathrm{~d} R_{1}\right) \cdots Q\left(\mathrm{~d} R_{k}\right)\right)^{1 / 2} \\
& \leq \sum_{k=0}^{A_{2}\left|\Lambda_{n}\right|} \frac{1}{k !}\left(z\left|\Lambda_{n}\right|\right)^{k}\left(Q^{\otimes A_{2}\left|\Lambda_{n}\right|}\left(\sum_{i=1}^{A_{2}\left|\Lambda_{n}\right|}\left(1+R_{i}^{2}\right)>A_{1}\left|\Lambda_{n}\right|\right)\right)^{1 / 2} \\
& \times\left(k^{2} \int\left(1+R^{2}\right)^{2} \mathrm{e}^{2 C\left(1+R^{2}\right)} Q(\mathrm{~d} R)\left(\int \mathrm{e}^{2 C\left(1+R^{2}\right)} Q(\mathrm{~d} R)\right)^{k-1}\right)^{1 / 2} .
\end{aligned}
$$

Therefore, thanks to assumption $(\mathrm{H})$, there exists a constant $B_{2}$ such that

$$
\begin{aligned}
I_{3} & \leq\left(Q^{\otimes A_{2}\left|\Lambda_{n}\right|}\left(\sum_{i=1}^{A_{2}\left|\Lambda_{n}\right|}\left(1+R_{i}^{2}\right)>A_{1}\left|\Lambda_{n}\right|\right)\right)^{1 / 2} \sum_{k=0}^{A_{2}\left|\Lambda_{n}\right|} \frac{1}{k !}\left(z\left|\Lambda_{n}\right|\right)^{k} B_{2}^{k} \\
& \leq\left(Q^{\otimes A_{2}\left|\Lambda_{n}\right|}\left(\sum_{i=1}^{A_{2}\left|\Lambda_{n}\right|}\left(1+R_{i}^{2}\right)>A_{1}\left|\Lambda_{n}\right|\right)\right)^{1 / 2} \exp \left(z B_{2}\left|\Lambda_{n}\right|\right) .
\end{aligned}
$$

By Lemma 3.1 we can choose $A_{1}$ such that

$$
Q^{\otimes A_{2}\left|\Lambda_{n}\right|}\left(\sum_{i=1}^{A_{2}\left|\Lambda_{n}\right|}\left(1+R_{i}^{2}\right)>A_{1}\left|\Lambda_{n}\right|\right) \leq \exp \left(-2 z B_{2}\left|\Lambda_{n}\right|\right)
$$

and so $I_{3} \leq 1$. This completes the proof.

We deduce from Proposition 3.3 and (3.2) that there exists a constant $A$ such that

$$
\mathcal{I}\left(\hat{\mu}_{n}\right) \leq A \text { for all } n \in \mathbb{N}^{*} .
$$

By Proposition 3.2, there exists a subsequence $\left(\hat{\mu}_{\varphi(n)}\right)$ which converges to a probability measure $\hat{\mu}$ for the topology $\tau_{W}$. To simplify the notation, we write $\left(\hat{\mu}_{n}\right)$ to denote $\left(\hat{\mu}_{\varphi(n)}\right)$. Let us remark that $\hat{\mu}$ is stationary under the translations $\left(\vartheta_{i}\right)_{i \in \mathbb{Z}^{2}}$ since it is the case for the measures $\left(\hat{\mu}_{n}\right)$. 


\subsection{Properties of the measures $\left(\hat{\mu}_{n}\right)$ and $\hat{\mu}$}

In the following lemma we prove that the support of the measure $\hat{\mu}$ is included in $\mathcal{M}_{\mathcal{T}}$.

Lemma 3.2. We have $\hat{\mu}\left(\mathcal{M}_{\mathcal{T}}\right)=1$.

Proof. Let us show that, for $\hat{\mu}$-almost every $\gamma$, there exist $K$ and $K^{\prime}$ such that (2.1) is satisfied. Let us begin with the first property in (2.1).

From the analogue of (3.3) for $\hat{\mu}_{n}$, there exists a constant $A$ such that $\int \sum_{(x, R) \in \gamma_{\Lambda_{n}}}(1+$ $\left.R^{2}\right) \hat{\mu}_{n}(\mathrm{~d} \gamma) \leq A\left|\Lambda_{n}\right|$. Since $\hat{\mu}_{n}$ is stationary under the translations $\left(\vartheta_{i}\right)_{i \in \mathbb{Z}^{2}}$, we deduce that $\int \sum_{(x, R) \in \gamma_{[0,1]^{2}}}\left(1+R^{2}\right) \hat{\mu}_{n}(\mathrm{~d} \gamma) \leq A$. By the definition of the topology $\tau_{\mathcal{W}}$ we obtain the same inequality for $\hat{\mu}$, i.e.

$$
\int \sum_{(x, R) \in \gamma_{[0,1]^{2}}}\left(1+R^{2}\right) \hat{\mu}(\mathrm{d} \gamma) \leq A .
$$

So, by the ergodic theorem of [10] we obtain, $\hat{\mu}$-almost surely,

$$
\lim _{n \rightarrow \infty} \frac{1}{\pi n^{2}} \sum_{(x, R) \in \gamma_{B(0, n)}}\left(1+R^{2}\right)=\mathrm{E}_{\hat{\mu}}\left(\sum_{(x, R) \in \gamma_{[0,1]^{2}}}\left(1+R^{2}\right) \mid g\right),
$$

where $g$ is the sigma-field of invariant sets. So, the supremum over $n \in \mathbb{N}^{*}$ of the quantities $\left(1 / \pi n^{2}\right) \sum_{(x, R) \in \gamma_{B(0, n)}}\left(1+R^{2}\right)$ is $\hat{\mu}$-almost surely finite. Therefore, for $\hat{\mu}$-almost every $\gamma$, there exists $K$ such that the first property in (2.1) is satisfied.

Concerning the second property in $(2.1)$, let $\gamma$ be in $\mathcal{M}$. For every $k=\left(k^{1}, k^{2}\right)$ in $\mathbb{Z}^{2}, D_{k}$ denotes the cube $\left[k^{1}, k^{1}+1\right] \times\left[k^{2}, k^{2}+1\right]$ and $R_{k}$ denotes the value $\max \left\{R,(x, R) \in \gamma_{D_{k}}\right\}$. Let $\left(k_{n}\right)_{n \in \mathbb{N}}$ be a sequence of distinct elements in $\mathbb{Z}^{2}$, and let $0<a<b$ be reals such that

$$
\text { an } \leq\left|k_{n}\right|^{2} \leq b n \quad \text { and } \bigcup_{n \in \mathbb{N}}\left\{k_{n}\right\}=\mathbb{Z}^{2} \quad \text { for all } n \in \mathbb{N} .
$$

It is not difficult to show that such a sequence exists. In fact, $\left(k_{n}\right)$ scans $\mathbb{Z}^{2}$ by a spiral around $(0,0)$. So, using the stationarity of $\hat{\mu}$ and (3.6),

$$
\begin{aligned}
\sum_{k \in \mathbb{Z}^{2}} \hat{\mu}\left(R_{k} \geq \frac{1}{2}|k|\right) & =\sum_{k \in \mathbb{Z}^{2}} \hat{\mu}\left(1+R_{k}^{2} \geq \frac{1}{4}|k|^{2}+1\right) \\
& \leq \sum_{k \in \mathbb{Z}^{2}} \hat{\mu}\left(\sum_{(x, R) \in \gamma_{D_{k}}}\left(1+R^{2}\right) \geq \frac{1}{4}|k|^{2}+1\right) \\
& \leq \sum_{n \in \mathbb{N}} \hat{\mu}\left(\sum_{(x, R) \in \gamma_{D_{k_{n}}}}\left(1+R^{2}\right) \geq \frac{1}{4}\left|k_{n}\right|^{2}+1\right) \\
& \leq \sum_{n \in \mathbb{N}} \hat{\mu}\left(\sum_{(x, R) \in \gamma_{D_{0}}}\left(1+R^{2}\right) \geq \frac{a n}{4}\right) \\
& =\sum_{n \in \mathbb{N}}(n+1) \hat{\mu}\left(n \leq \frac{4}{a} \sum_{(x, R) \in \gamma_{D_{0}}}\left(1+R^{2}\right)<n+1\right) \\
& \left.\leq 1+\frac{4}{a} \mathrm{E}_{\hat{\mu}} \sum_{(x, R) \in \gamma_{D_{0}}} 1+R^{2}\right) .
\end{aligned}
$$


By (3.5), the last term in (3.7) is finite. Therefore, using the Borel-Cantelli lemma, there is $\hat{\mu}$-almost surely a finite number of indexes $k \in \mathbb{Z}^{2}$ such that $R_{k} \geq \frac{1}{2}|k|$. So, $\hat{\mu}$-almost surely, the second property in (2.1) is satisfied for large enough $K^{\prime}$. This completes the proof.

In the following section, to prove that $\hat{\mu}$ satisfies the DLR equations, we need some uniform property for the supports of the measures $\left(\hat{\mu}_{n}\right)$. We would like to prove that, for every $\varepsilon>0$, there exists $K$ and $K^{\prime}$ such that, for all $n \geq 1, \hat{\mu}_{n}\left(\mathcal{M}_{K, K^{\prime}}\right) \geq 1-\varepsilon$. We have not been able to prove this, but we have proved the following weak property which will be sufficient.

Lemma 3.3. For every $\varepsilon>0$, there exists a $K^{\prime}$ such that, for all $n \geq 1$,

$$
\hat{\mu}_{n}\left(\mathcal{M}_{K^{\prime}}\right) \geq 1-\varepsilon
$$

where $\mathcal{M}_{K^{\prime}}$ is the set of $\gamma \in \mathcal{M}$ such that, for all $n \geq 1$,

$$
\sup _{(x, R) \in \gamma_{B(0, n)}} R \leq \frac{1}{2} n+K^{\prime} .
$$

Before giving the proof, we need the following lemma.

Lemma 3.4. ([4, Lemma 5.2].) For every $\alpha \geq 0$, every $\varepsilon>0$, and every $\Delta$ in $\mathscr{B}\left(\mathbb{R}^{2}\right)$, there exists a constant $\beta$ such that, for every probability measure $\mathrm{P}$ on $\mathcal{M}$ such that $\mathcal{I}(\mathrm{P}) \leq \alpha$,

$$
\mathrm{E}_{\mathrm{P}}\left(\mathbf{1}_{\left\{\sum_{(x, R) \in \gamma_{\Delta}}\left(1+R^{2}\right) \geq \beta\right\}} \sum_{(x, R) \in \gamma_{\Delta}}\left(1+R^{2}\right)\right) \leq \varepsilon .
$$

Proof of Lemma 3.3. Let $\varepsilon>0$ and $c>\sqrt{b}$, which we will fix later. For every $n \geq 1$, following a similar calculation to (3.7),

$$
\begin{aligned}
& \sum_{k \in \mathbb{Z}^{2} \backslash B(o, c)} \hat{\mu}_{n}\left(R_{k} \geq \frac{1}{2}|k|\right) \\
& \leq \sum_{n \geq c^{2} / b} \hat{\mu}_{n}\left(\sum_{(x, R) \in \gamma_{D_{0}}}\left(1+R^{2}\right) \geq \frac{a n}{4}\right) \\
& \leq \mathrm{E}_{\hat{\mu}_{n}}\left(\mathbf{1}_{\left\{(4 / a) \sum_{(x, R) \in \gamma_{D_{0}}}\left(1+R^{2}\right) \geq c^{2} / b\right\}}\left(1-\frac{c^{2}}{b}+\frac{4}{a} \sum_{(x, R) \in \gamma_{D_{0}}} 1+R^{2}\right)\right) \\
& \leq \frac{4}{a} \mathrm{E}_{\hat{\mu}_{n}}\left(\mathbf{1}_{\left\{\sum_{(x, R) \in \gamma_{D_{0}}}\left(1+R^{2}\right) \geq a c^{2} / 4 b\right\}}\left(\sum_{(x, R) \in \gamma_{D_{0}}} 1+R^{2}\right)\right) .
\end{aligned}
$$

Thanks to property (3.4) and Lemma 3.4, we deduce that, for large enough $c$, inequality (3.9) becomes

$$
\sum_{k \in \mathbb{Z}^{2} \backslash B(0, c)} \hat{\mu}_{n}\left(R_{k} \geq \frac{1}{2}|k|\right) \leq \frac{\varepsilon}{2} .
$$

So, for all $n \geq 1$,

$$
\hat{\mu}_{n}\left(\sup _{k \in \mathbb{Z}^{2} \backslash B(0, c)} \frac{R_{k}}{|k|} \geq \frac{1}{2}\right) \leq \frac{\varepsilon}{2} .
$$


Moreover, we can again apply Lemma 3.4 to prove that there exists a $K^{\prime}$ large enough such that, for all $n \geq 1$,

$$
\hat{\mu}_{n}\left(\sum_{(x, R) \in \gamma_{B(0, c)}}\left(1+R^{2}\right) \geq K^{\prime}\right) \leq \frac{\varepsilon}{2},
$$

and so

$$
\hat{\mu}_{n}\left(\sup _{k \in B(0, c)} R_{k} \geq K^{\prime}\right) \leq \frac{\varepsilon}{2}
$$

Combining inequalities (3.10) and (3.11) we obtain, for all $n \geq 1, \hat{\mu}_{n}\left(\mathcal{M}_{K^{\prime}}^{c}\right) \leq \varepsilon$.

\subsection{The DLR equations}

In this section we prove that $\hat{\mu}$ satisfies the DLR equations, (2.3), for every $\Lambda$ in $\mathcal{B}\left(\mathbb{R}^{2}\right)$. Let $f$ be a local bounded measurable function from $\mathcal{M}_{\mathcal{T}}$ to $\mathbb{R}$. Then $f_{\Lambda}$ denotes the following function

$$
\begin{aligned}
f_{\Lambda}: \mathcal{M}_{\mathcal{T}} & \rightarrow \mathbb{R}, \\
\gamma & \mapsto \int f\left(\gamma^{\prime}\right) \Xi_{\Lambda}\left(\gamma, \mathrm{d} \gamma^{\prime}\right),
\end{aligned}
$$

and (2.3) for $\Lambda$ and $f$ becomes

$$
\int f \mathrm{~d} \hat{\mu}=\int f_{\Lambda} \mathrm{d} \hat{\mu}
$$

Let us remark that the function $f_{\Lambda}$ is not local even if $f$ is. Indeed, the kernel $\Xi_{\Lambda}$ is not local since the support of $Q$ is a priori not bounded and so some far-removed points may contribute to the energy $H_{\Lambda}$ in $\Lambda$.

It is easy to see that the measures $\left(\hat{\mu}_{n}\right)$ do not satisfy the DLR equations. So, let us introduce a family of measures $\left(\bar{\mu}_{n}\right)$, asymptotically equivalent to $\left(\hat{\mu}_{n}\right)$ for the topology $\tau_{w}$, which satisfy the DLR equations.

For every $n \geq 1$, we set

$$
\bar{\mu}_{n}=\frac{1}{(2 n+1)^{2}} \sum_{\substack{i \in I_{n} \\ \Lambda \subset \vartheta_{i}\left(\Lambda_{n}\right)}} \mu_{n} \circ \vartheta_{i}^{-1} .
$$

Let us remark that $\bar{\mu}_{n}$ is not necessarily a probability measure since the sum is not over all $i$ in $I_{n}$. Let us show that $\bar{\mu}_{n}$ satisfies the DLR equation for $\Lambda$. First of all, it is obvious that $\mu_{n}$ satisfies the DLR equation if the set $\Lambda$ is included in $\Lambda_{n}$. Indeed, it is just the compatibility of the kernels $\left(\Xi_{\Lambda}\right)_{\Lambda \in \mathscr{B}\left(\mathbb{R}^{2}\right)}$ resulting from the additivity of the energy (see Lemma 2.2). So,

$$
\begin{aligned}
\int f_{\Lambda}(\gamma) \bar{\mu}_{n}(\mathrm{~d} \gamma) & =\frac{1}{(2 n+1)^{2}} \sum_{\substack{i \in I_{n} \\
\Lambda \subset \vartheta_{i}\left(\Lambda_{n}\right)}} \int f\left(\gamma^{\prime}\right) \Xi_{\Lambda}\left(\gamma, \mathrm{d} \gamma^{\prime}\right) \mu_{n} \circ \vartheta_{i}^{-1}(\mathrm{~d} \gamma) \\
& =\frac{1}{(2 n+1)^{2}} \sum_{\substack{i \in I_{n} \\
\Lambda \subset \vartheta_{i}\left(\Lambda_{n}\right)}} \int f\left(\vartheta_{i}\left(\gamma^{\prime}\right)\right) \Xi_{\vartheta_{i}^{-1}(\Lambda)}\left(\gamma, \mathrm{d} \gamma^{\prime}\right) \mu_{n}(\mathrm{~d} \gamma) \\
& =\frac{1}{(2 n+1)^{2}} \sum_{\substack{i \in I_{n} \\
\Lambda \subset \vartheta_{i}\left(\Lambda_{n}\right)}} \int f\left(\vartheta_{i}(\gamma)\right) \mu_{n}(\mathrm{~d} \gamma) \\
& =\int f(\gamma) \bar{\mu}_{n}(\mathrm{~d} \gamma) .
\end{aligned}
$$


Now, we need to prove that $\left(\bar{\mu}_{n}\right)$ converges to $\hat{\mu}$ for the topology $\tau_{w}$. The following lemma, for which the proof is almost the same as the one given in [4], ensures that $\left(\hat{\mu}_{n}\right)$ and $\left(\bar{\mu}_{n}\right)$ are asymptotically equivalent for the topology $\tau_{W}$.

Lemma 3.5. For every local and tame function $f$ in $\mathrm{W}$,

$$
\lim _{n \rightarrow+\infty}\left|\int f \mathrm{~d} \bar{\mu}_{n}-\int f \mathrm{~d} \hat{\mu}_{n}\right|=0 .
$$

Proof. Let $f$ be a local and tame function in $\mathcal{W}$. We denote by $\Delta$ its support, and there exists $b>0$ such that, for every $\gamma \in \mathcal{M}$,

$$
|f(\gamma)| \leq b\left(1+\sum_{(x, R) \in \gamma_{\Delta}} 1+R^{2}\right) .
$$

We choose $n$ large enough such that $\Delta$ and $\Lambda$ are included in $\Lambda_{n}$. So the quantity $\delta=$ $\left|\int f \mathrm{~d} \bar{\mu}_{n}-\int f \mathrm{~d} \hat{\mu}_{n}\right|$ satisfies

$$
\begin{aligned}
\delta & =\left|\frac{1}{(2 n+1)^{2}} \sum_{\substack{i \in I_{n} \\
\Lambda \subset \vartheta_{i}\left(\Lambda_{n}\right)}} \int f(\gamma) \mu_{n} \circ \vartheta_{i}^{-1}(\mathrm{~d} \gamma)-\frac{1}{(2 n+1)^{2}} \sum_{i \in I_{n}} \int f(\gamma) \tilde{\mu}_{n} \circ \vartheta_{i}^{-1}(\mathrm{~d} \gamma)\right| \\
& \leq \frac{1}{(2 n+1)^{2}} \sum_{\substack{i \in I_{n} \\
\Lambda \cup \Delta \nsubseteq \vartheta_{i}\left(\Lambda_{n}\right)}}\left|\int f(\gamma) \tilde{\mu}_{n} \circ \vartheta_{i}^{-1}(\mathrm{~d} \gamma)\right| .
\end{aligned}
$$

Since $\Lambda \cup \Delta$ is bounded, there exists $k>0$ such that $\Lambda \cup \Delta \subset[-k, k]^{2}$. So,

$$
\operatorname{card}\left(i \in I_{n} \text { such that } \Lambda \cup \Delta \nsubseteq \vartheta_{i}\left(\Lambda_{n}\right)\right) \leq 4 k(2 n+1) .
$$

Therefore, for any $a>0$,

$$
\begin{aligned}
\delta \leq & \frac{b}{(2 n+1)^{2}} \sum_{\substack{i \in I_{n} \\
\Lambda \cup \Delta \nsubseteq \vartheta_{i}\left(\Lambda_{n}\right)}} \int\left(1+\sum_{(x, R) \in \gamma_{\Delta}} 1+R^{2}\right) \tilde{\mu}_{n} \circ \vartheta_{i}^{-1}(\mathrm{~d} \gamma) \\
\leq & \frac{b}{(2 n+1)^{2}} \sum_{\substack{i \in I_{n} \\
\Lambda \cup \Delta \vartheta_{i}\left(\Lambda_{n}\right)}} \int \mathbf{1}_{\left\{\sum_{(x, R) \in \gamma_{\Delta}} 1+R^{2} \geq a\right\}}\left(\sum_{(x, R) \in \gamma_{\Delta}} 1+R^{2}\right) \tilde{\mu}_{n} \circ \vartheta_{i}^{-1}(\mathrm{~d} \gamma) \\
& +\frac{4 a b k(2 n+1)}{(2 n+1)^{2}}+\frac{4 k b(2 n+1)}{(2 n+1)^{2}} \\
\leq & \left.b \int \mathbf{1}_{\left\{\sum_{(x, R) \in \gamma_{\Delta}}\right.} 1+R^{2} \geq a\right\}\left(\sum_{(x, R) \in \gamma_{\Delta}} 1+R^{2}\right) \hat{\mu}_{n}(\mathrm{~d} \gamma)+\frac{4(a+1) b}{2 n+1} .
\end{aligned}
$$

Let, $\varepsilon>0$. By Lemma 3.4, the first term in (3.15) is, for large enough $a$ and every $n$ in $\mathbb{N}^{*}$, smaller than $\varepsilon / 2$. The second term is, for large enough $n$, smaller than $\varepsilon / 2$ too. Therefore, for large enough $n, \delta$ is smaller than $\varepsilon$ and the lemma is proved.

We have proved that $\left(\bar{\mu}_{n}\right)$ converges to $\hat{\mu}$ and that, for every $n \geq 1, \bar{\mu}_{n}$ satisfies DLR equation (3.12). So we would like to pass the limit through these equations to get the DLR 
equations for $\hat{\mu}$, but this is not possible because the function $f_{\Lambda}$ is not local. Let us use some approximation methods similar as the ones used by [11]. Let $\varepsilon>0$. Our aim is to prove that $\delta=\left|\int f_{\Lambda}(\gamma) \hat{\mu}(\mathrm{d} \gamma)-\int f(\gamma) \hat{\mu}(\mathrm{d} \gamma)\right| \leq \varepsilon$, which implies that $\delta=0$ and so the DLR equation (3.12) is satisfied. We set $M>0$ and

$$
A_{\Lambda}^{M}=\left\{\gamma \in \mathcal{M} \text { such that, for all }(x, R) \in \gamma_{\Lambda}, R \leq M\right\} .
$$

By Lemmas 3.2 and 3.3, there exists $C_{0}>0$ such that, for all $K \geq C_{0}, K^{\prime} \geq C_{0}, M \geq C_{0}$, and all $n \geq 1$,

$$
\hat{\mu}\left(\mathcal{M}_{K, K^{\prime}}\right) \geq 1-\varepsilon, \quad \hat{\mu}_{n}\left(\mathcal{M}_{K^{\prime}}\right) \geq 1-\varepsilon, \quad \text { and } \quad \hat{\mu}_{n}\left(A_{\Lambda}^{M}\right) \geq 1-\varepsilon .
$$

From definitions of $\left(\bar{\mu}_{n}\right)$ and $\left(\hat{\mu}_{n}\right)$ we easily obtain

$$
\bar{\mu}_{n}\left(\mathcal{M}_{K^{\prime}}^{c}\right) \leq \hat{\mu}_{n}\left(\mathcal{M}_{K^{\prime}}^{c}\right)
$$

and

$$
\bar{\mu}_{n}\left(\left(A_{\Lambda}^{M}\right)^{c}\right) \leq \hat{\mu}_{n}\left(\left(A_{\Lambda}^{M}\right)^{c}\right)
$$

So (3.16) becomes

$$
\hat{\mu}\left(\mathcal{M}_{K, K^{\prime}}\right) \geq 1-\varepsilon, \quad \bar{\mu}_{n}\left(\mathcal{M}_{K^{\prime}}\right) \geq 1-\varepsilon, \quad \text { and } \quad \bar{\mu}_{n}\left(A_{\Lambda}^{M}\right) \geq 1-\varepsilon .
$$

Throughout the remainder of the paper, $K$ and $K^{\prime}$ are fixed constants larger than $C_{0}$. The constant $M$ is larger than $C_{0}$ too and it will be fixed later. Moreover, via a rescaling procedure, we can suppose that $\|f\|_{\infty} \leq 1$. Therefore,

$$
\begin{aligned}
\delta & \leq\|f\|_{\infty} \hat{\mu}\left(\mathcal{M}_{K, K^{\prime}}^{c}\right)+\left|\int_{\mathcal{M}_{K, K^{\prime}}} f_{\Lambda}(\gamma) \hat{\mu}(\mathrm{d} \gamma)-\int f(\gamma) \hat{\mu}(\mathrm{d} \gamma)\right| \\
& \leq \varepsilon+\left|\int_{\mathcal{M}_{K, K^{\prime}}} f_{\Lambda}(\gamma) \hat{\mu}(\mathrm{d} \gamma)-\int f(\gamma) \hat{\mu}(\mathrm{d} \gamma)\right| .
\end{aligned}
$$

Let $\Lambda^{\prime}$ be a bounded set in $\mathscr{B}\left(\mathbb{R}^{2}\right)$ such that $\Lambda$ and the support of $f$ are included in $\Lambda^{\prime}$. Then we define the function $g_{\Lambda^{\prime}}^{M}$ from $\mathcal{M}$ to $\mathbb{R}$ by

$$
g_{\Lambda^{\prime}}^{M}(\gamma)=\int \mathbf{1}_{A_{\Lambda}^{M}}\left(\gamma_{\Lambda}^{\prime}\right) f\left(\gamma_{\Lambda}^{\prime}+\gamma_{\Lambda^{\prime} \backslash \Lambda}\right) \frac{1}{Z_{\Lambda}^{M}\left(\gamma_{\Lambda^{\prime} \backslash \Lambda}\right)} \exp \left(-H_{\Lambda}\left(\gamma_{\Lambda}^{\prime}+\gamma_{\Lambda^{\prime} \backslash \Lambda}\right)\right) \pi_{\Lambda}\left(\mathrm{d} \gamma_{\Lambda}^{\prime}\right),
$$

where

$$
Z_{\Lambda}^{M}\left(\gamma_{\Lambda^{\prime} \backslash \Lambda}\right)=\int \mathbf{1}_{A_{\Lambda}^{M}}\left(\gamma_{\Lambda}^{\prime}\right) \exp \left(-H_{\Lambda}\left(\gamma_{\Lambda}^{\prime}+\gamma_{\Lambda^{\prime} \backslash \Lambda}\right)\right) \pi_{\Lambda}\left(\mathrm{d} \gamma_{\Lambda}^{\prime}\right)
$$

The function $g_{\Lambda^{\prime}}^{M}$ is a local modification of the function $f_{\Lambda}$. More precisely, we have the following lemma.

Lemma 3.6. For large enough $M$ and $\Lambda^{\prime}$,

$$
\sup _{\gamma \in \mathcal{M}_{K, K^{\prime}}}\left|f_{\Lambda}(\gamma)-g_{\Lambda^{\prime}}^{M}(\gamma)\right| \leq \varepsilon
$$


Proof. Let us begin by dealing with the functions $Z_{\Lambda}^{M}$ and $Z_{\Lambda}$. By Lemmas 2.1 and 2.4, for every $\gamma$ in $\mathcal{M}_{K, K^{\prime}}$,

$$
\begin{aligned}
& \left|Z_{\Lambda}^{M}\left(\gamma_{\Lambda^{\prime} \backslash \Lambda}\right)-Z_{\Lambda}\left(\gamma_{\Lambda^{\mathrm{c}}}\right)\right| \\
& \quad \leq \int \mathbf{1}_{\left\{B\left(0,2\left(R_{1}+\bar{R}+K^{\prime}+1\right)\right) \nsubseteq \subseteq \Lambda^{\prime}\right\} \cup\left\{\gamma_{\Lambda}^{\prime} \notin A_{\Lambda}^{M}\right\}} \exp \left(C^{\prime} \sum_{(x, R) \in \gamma_{\Lambda}^{\prime}} 1+R^{2}\right) \pi_{\Lambda}\left(\mathrm{d} \gamma_{\Lambda}^{\prime}\right),
\end{aligned}
$$

where $R_{1}$ is a real such that $\Lambda \subset B\left(0, R_{1}\right)$ and $\bar{R}=\sup \left\{R,(x, R) \in \gamma_{\Lambda}^{\prime}\right\}$. The right-hand side term does not depend on $\gamma$ and, thanks to the dominated convergence theorem (the assumptions are satisfied via (H)), it goes to 0 when $M$ goes to $\infty$ and $\Lambda^{\prime}$ goes to $\mathbb{R}^{2}$. So the application $\gamma \mapsto Z_{\Lambda}^{M}\left(\gamma_{\Lambda^{\prime} \backslash \Lambda}\right)-Z_{\Lambda}\left(\gamma_{\Lambda^{c}}\right)$ converges uniformly on $\mathcal{M}_{K, K^{\prime}}$ to 0 when $M$ goes to $\infty$ and $\Lambda^{\prime}$ goes to $\mathbb{R}^{2}$.

Similarly, we show that the application

$$
\begin{aligned}
\gamma \mapsto & \int \mathbf{1}_{A_{\Lambda}^{M}}\left(\gamma_{\Lambda}^{\prime}\right) f\left(\gamma_{\Lambda}^{\prime}+\gamma_{\Lambda^{\prime} \backslash \Lambda}\right) \exp \left(-H_{\Lambda}\left(\gamma_{\Lambda}^{\prime}+\gamma_{\Lambda^{\prime} \backslash \Lambda}\right)\right) \pi_{\Lambda}\left(\mathrm{d} \gamma_{\Lambda}^{\prime}\right) \\
& -\int f\left(\gamma_{\Lambda}^{\prime}+\gamma_{\Lambda^{c}}\right) \exp \left(-H_{\Lambda}\left(\gamma_{\Lambda}^{\prime}+\gamma_{\Lambda^{c}}\right)\right) \pi_{\Lambda}\left(\mathrm{d} \gamma_{\Lambda}^{\prime}\right)
\end{aligned}
$$

converges uniformly on $\mathcal{M}_{K, K^{\prime}}$ to 0 when $M$ goes to $\infty$ and $\Lambda^{\prime}$ goes to $\mathbb{R}^{2}$. Since $Z_{\Lambda}$ and $Z_{\Lambda}^{M}$ are bigger than $\pi_{\Lambda}(\varnothing)=\mathrm{e}^{-z|\Lambda|}$, we deduce that $g_{\Lambda^{\prime}}^{M}$ converges uniformly on $\mathcal{M}_{K, K^{\prime}}$ to $f_{\Lambda}$. Lemma 3.6 is proved.

We now fix $\Lambda^{\prime}$ and $M$ such that the assumptions of Lemma 3.6 are satisfied, and we suppose for later that

$$
B\left(0,2\left(R_{1}+M+K^{\prime}+1\right)\right) \subset \Lambda^{\prime} .
$$

Inequality (3.18) becomes

$$
\begin{aligned}
\delta & \leq 2 \varepsilon+\left|\int_{\mathcal{M}_{K, K^{\prime}}} g_{\Lambda^{\prime}}^{M}(\gamma) \hat{\mu}(\mathrm{d} \gamma)-\int f(\gamma) \hat{\mu}(\mathrm{d} \gamma)\right| \\
& \leq 3 \varepsilon+\left|\int g_{\Lambda^{\prime}}^{M}(\gamma) \hat{\mu}(\mathrm{d} \gamma)-\int f(\gamma) \hat{\mu}(\mathrm{d} \gamma)\right| .
\end{aligned}
$$

The function $g_{\Lambda^{\prime}}^{M}$ is obviously local and bounded by $\|f\|_{\infty} \leq 1$, and since the measures $\left(\bar{\mu}_{n}\right)$ converge to $\hat{\mu}$ for the topology $\tau_{\mathcal{W}}$, we fix $n$ large enough such that

$$
\left|\int g_{\Lambda^{\prime}}^{M}(\gamma) \hat{\mu}(\mathrm{d} \gamma)-\int g_{\Lambda^{\prime}}^{M}(\gamma) \bar{\mu}_{n}(\mathrm{~d} \gamma)\right| \leq \varepsilon \quad \text { and } \quad\left|\int f(\gamma) \hat{\mu}(\mathrm{d} \gamma)-\int f(\gamma) \bar{\mu}_{n}(\mathrm{~d} \gamma)\right| \leq \varepsilon .
$$

Therefore, from (3.17) and (3.20),

$$
\begin{aligned}
\delta & \leq 5 \varepsilon+\left|\int g_{\Lambda^{\prime}}^{M}(\gamma) \bar{\mu}_{n}(\mathrm{~d} \gamma)-\int f(\gamma) \bar{\mu}_{n}(\mathrm{~d} \gamma)\right| \\
& \leq 6 \varepsilon+\left|\int_{\mathcal{M}_{K^{\prime}}} g_{\Lambda^{\prime}}^{M}(\gamma) \bar{\mu}_{n}(\mathrm{~d} \gamma)-\int f(\gamma) \bar{\mu}_{n}(\mathrm{~d} \gamma)\right| .
\end{aligned}
$$

To finish, we need the following lemma. 
Lemma 3.7. We have

$$
\left|\int_{\mathcal{M}_{K^{\prime}}} f_{\Lambda}(\gamma) \bar{\mu}_{n}(\mathrm{~d} \gamma)-\int_{\mathcal{M}_{K^{\prime}}} g_{\Lambda^{\prime}}^{M}(\gamma) \bar{\mu}_{n}(\mathrm{~d} \gamma)\right| \leq 2 \varepsilon .
$$

Proof. Thanks to a simple conditional calculus,

$$
\begin{aligned}
\mid \int_{\mathcal{M}_{K^{\prime}}} & f_{\Lambda}(\gamma) \bar{\mu}_{n}(\mathrm{~d} \gamma)-\int_{\mathcal{M}_{K^{\prime}}} g_{\Lambda^{\prime}}^{M}(\gamma) \bar{\mu}_{n}(\mathrm{~d} \gamma) \mid \\
= & \mid \int_{\mathcal{M}_{K^{\prime}}} f\left(\gamma^{\prime}\right) \Xi_{\Lambda}\left(\gamma, \mathrm{d} \gamma^{\prime} \mid \gamma^{\prime} \in A_{\Lambda}^{M}\right)\left(1-\Xi_{\Lambda}\left(\gamma,\left(A_{\Lambda}^{M}\right)^{c}\right)\right) \bar{\mu}_{n}(\mathrm{~d} \gamma) \\
& +\int_{\mathcal{M}_{K^{\prime}}} f\left(\gamma^{\prime}\right) \Xi_{\Lambda}\left(\gamma, \mathrm{d} \gamma^{\prime} \mid \gamma^{\prime} \notin A_{\Lambda}^{M}\right) \Xi_{\Lambda}\left(\gamma,\left(A_{\Lambda}^{M}\right)^{c}\right) \bar{\mu}_{n}(\mathrm{~d} \gamma) \\
& -\int_{\mathcal{M}_{K^{\prime}}} g_{\Lambda^{\prime}}^{M}(\gamma) \bar{\mu}_{n}(\mathrm{~d} \gamma) \mid \\
\leq & 2\|f\|_{\infty} \int \Xi_{\Lambda}\left(\gamma,\left(A_{\Lambda}^{M}\right)^{c}\right) \bar{\mu}_{n}(\mathrm{~d} \gamma) \\
& +\left|\int_{\mathcal{M}_{K^{\prime}}} f\left(\gamma^{\prime}\right) \Xi_{\Lambda}\left(\gamma, \mathrm{d} \gamma^{\prime} \mid \gamma^{\prime} \in A_{\Lambda}^{M}\right) \bar{\mu}_{n}(\mathrm{~d} \gamma)-\int_{\mathcal{M}_{K^{\prime}}} g_{\Lambda^{\prime}}^{M}(\gamma) \bar{\mu}_{n}(\mathrm{~d} \gamma)\right| .
\end{aligned}
$$

We remark easily that the last term in (3.21) is null since, by Lemma 2.1 and property (3.19), the two kernels are equal on $\mathcal{M}_{K^{\prime}}$. The measure $\bar{\mu}_{n}$ satisfies DLR equation (3.14), so $\int \Xi_{\Lambda}\left(\gamma,\left(A_{\Lambda}^{M}\right)^{c}\right) \bar{\mu}_{n}(\mathrm{~d} \gamma)=\bar{\mu}_{n}\left(\left(A_{\Lambda}^{M}\right)^{c}\right)$, and thanks to (3.17), the lemma is proved.

By Lemma 3.7 and properties (3.17),

$$
\begin{aligned}
\delta & \leq 8 \varepsilon+\left|\int_{\mathcal{M}_{K^{\prime}}} f_{\Lambda}(\gamma) \bar{\mu}_{n}(\mathrm{~d} \gamma)-\int f(\gamma) \bar{\mu}_{n}(\mathrm{~d} \gamma)\right| \\
& \leq 9 \varepsilon+\left|\int f_{\Lambda}(\gamma) \bar{\mu}_{n}(\mathrm{~d} \gamma)-\int f(\gamma) \bar{\mu}_{n}(\mathrm{~d} \gamma)\right| .
\end{aligned}
$$

The last term in (3.22) is null since $\bar{\mu}_{n}$ satisfies the DLR equation (3.14) for $\Lambda$. Therefore, $\delta \leq 9 \varepsilon$ and so $\delta=0$. The DLR equations (2.3) are satisfied for $\hat{\mu}$ and any $\Lambda$ in $\mathscr{B}\left(\mathbb{R}^{2}\right)$.

\section{Concluding remarks}

To conclude this paper, we would like to give some comments about the possible extensions of Theorem 2.1. First of all, concerning the dimension of the space $\mathbb{R}^{d}$, if $d>2$, there is no a priori problem for the general scheme of the proof. We just have to check that the energy function $H$ is stable, and so some conditions may appear. For example, the Euler-Poincaré characteristic $\chi$ is not stable in dimension 3. Similarly, we think it is possible to apply our method to the case of general convex grains, which would not be balls, if the energy functions remain stable.

Now, concerning the form of the interaction $H$, we think it is possible to extend our result to some nonadditive functionals as long as the interaction remains local. Indeed, we do not really use the additive property of the Minkovski functional, except in the justification of Definition 2.2. A finite-range property would seem to be sufficient. Let us point out that important problems 
may appear if the interaction is not local. We have tried to prove the existence of a Gibbs germgrain model for nonlocal interaction, proposed by Møller and Helisova [9], which is equal to the number of connected components. But, in this case, some dependence with 'infinity' appears if there is percolation, and so we are not able to prove that the limiting measure $\hat{\mu}$ satisfies the DLR equations. It is a very interesting problem that remains open.

\section{References}

[1] Baddeley, A. J. And van Lieshout, M. N. M. (1995). Area-interaction point processes. Ann. Inst. Statist. Math. 47, 601-619.

[2] GeorgiI, H.-O. (1979). Canonical Gibbs Measure (Lecture Notes Math. 760). Springer, Berlin.

[3] Georgit, H.-O. And HägGström, O. (1996). Phase transition in continuum Potts models. Commun. Math. Phys. 181, 507-528.

[4] Georgit, H.-O. and Zessin, H. (1993). Large deviations and the maximum entropy principle for marked point random fields. Prob. Theory Relat. Fields 96, 177-204.

[5] Kendall, W. S., van Lieshout, M. N. M. and Baddeley, A. J. (1999). Quermass-interaction processes: conditions for stability. Adv. Appl. Prob. 31, 315-342.

[6] Likos, C. N., Mecke, K. R. And Wagner, H. (1995). Statistical morphology of random interface microemulsions. J. Chem. Phys. 102, 9350-9361.

[7] Mecke, K. R. (1996). A morphological model for complex fluids. J. Phys. Condens. Matter 8, $9663-9667$.

[8] Molchanov, I. (1997). Statistics of the Boolean Model for Practitioners and Mathematicians. John Wiley, Chichester.

[9] Møller, J. and Helisovì, K. (2008). Power diagrams and interaction processes for unions of discs. Adv. Appl. Prob. 40, 321-347.

[10] Nguyen, X.-X. And Zessin, H. (1979). Ergodic theorems for spatial processes. Z. Wahrscheinlichkeitsth. 48, 133-158.

[11] Preston, C. (1976). Random Fields (Lecture Notes Math. 534). Springer, Berlin.

[12] Ruelle, D. (1970). Superstable interactions in classical statistical mechanics. Commun. Math. Phys. 18, 127-159.

[13] Stoyan, D., Kendall, W. S. and Mecke, J. (1987). Stochastic Geometry and Its Applications. John Wiley, Chichester.

[14] Widom, B. ANd Rowlinson, J. S. (1970). New model for the study of liquid-vapor phase transitions. J. Chem. Phys. 52, 1670-1684. 